\title{
Evolution of metabolic divergence in Pseudomonas aeruginosa during long-term infection facilitates a proto-cooperative interspecies interaction
}

\author{
Charlotte Frydenlund Michelsen ${ }^{1}$, Seyed Mohammad Hossein Khademi ${ }^{1}$, \\ Helle Krogh Johansen ${ }^{2,3}$, Hanne Ingmer ${ }^{4}$, Pieter C Dorrestein ${ }^{5,6,7}$ and Lars Jelsbak ${ }^{1}$ \\ ${ }^{1}$ Department of Systems Biology, Technical University of Denmark, Lyngby, Denmark; ${ }^{2}$ Department of Clinical \\ Microbiology, Rigshospitalet, Copenhagen, Denmark; ${ }^{3}$ The Novo Nordisk Foundation Center for \\ Biosustainability, Technical University of Denmark, Hørsholm, Denmark; ${ }^{4}$ Department of Veterinary Disease \\ Biology, Food Safety and Zoonoses, University of Copenhagen, Frederiksberg C, Denmark; ${ }^{5}$ Department of \\ Pharmacology, University of California at San Diego, La Jolla, CA, USA; ${ }^{6}$ Department of Chemistry and \\ Biochemistry, University of California at San Diego, La Jolla, CA, USA and ${ }^{7}$ Skaggs School of Pharmacy and \\ Pharmaceutical Sciences, University of California at San Diego, La Jolla, CA, USA
}

\begin{abstract}
The effect of polymicrobial interactions on pathogen physiology and how it can act either to limit pathogen colonization or to potentiate pathogen expansion and virulence are not well understood. Pseudomonas aeruginosa and Staphylococcus aureus are opportunistic pathogens commonly found together in polymicrobial human infections. However, we have previously shown that the interactions between these two bacterial species are strain dependent. Whereas $\boldsymbol{P}$. aeruginosa PA01, a commonly used laboratory strain, effectively suppressed $S$. aureus growth, we observed a commensal-like interaction between the human host-adapted strain, DK2-P2M24-2003, and S. aureus. In this study, characterization by matrix-assisted laser desorption ionization-time of flight (MALDI-TOF) imaging mass spectrometry (IMS) and mass spectral (MS) molecular networking revealed a significant metabolic divergence between $P$. aeruginosa PAO1 and DK2-P2M24-2003, which comprised several virulence factors and signaling 4-hydroxy-2-alkylquinoline (HAQ) molecules. Strikingly, a further modulation of the HAQ profile was observed in DK2-P2M24-2003 during interaction with S. aureus, resulting in an area with thickened colony morphology at the $P$. aeruginosa-S. aureus interface. In addition, we found an HAQ-mediated protection of S. aureus by DK2-P2M24-2003 from the killing effect of tobramycin. Our findings suggest a model where the metabolic divergence manifested in human host-adapted $P$. aeruginosa is further modulated during interaction with $S$. aureus and facilitate a proto-cooperative $\boldsymbol{P}$. aeruginosa-S. aureus relationship.

The ISME Journal (2016) 10, 1323-1336; doi:10.1038/ismej.2015.220; published online 18 December 2015
\end{abstract}

\section{Introduction}

Although many bacterial infectious diseases are assumed to be monoclonal, infections most often occur in a polymicrobial context within the host (Short et al., 2014). How the different microbes contribute to disease and health remains poorly understood, but it is becoming increasingly clear that interactions between bacterial pathogens and other microbial species residing in the infected host (such as co-infecting pathogens or commensal bacteria) may in some cases determine disease phenotype or the response to therapy (Peters et al., 2012; Short et al., 2014). It continues to be a central challenge to

Correspondence: L Jelsbak, Department of Systems Biology, Technical University of Denmark, Building 301, Lyngby 2800, Denmark.

E-mail: lj@bio.dtu.dk

Received 15 May 2015; revised 20 October 2015; accepted 25 October 2015; published online 18 December 2015 identify and characterize these interactions and the biological processes they control in relation to infection. Studies of microbe-microbe interactions and their impact on the activity of bacterial pathogens have often focused on well-characterized laboratory or reference strains (Moree et al., 2012; Korgaonkar et al., 2013; Vega et al., 2013). However, given the genetic diversity that often exists in pathogen populations and the clonal diversity that may evolve within the host during long-term infection (Lieberman et al., 2011; Marvig et al., 2013; Markussen et al., 2014), a major issue is how well the use of any one strain as an experimental model for understanding how microbial interactions modulate pathogen activity reflects this diversity.

To begin to address this issue, we investigate chronic airway infections in patients with cystic fibrosis (CF). Complex microbial communities reside in the CF airways, where the community structure and composition may vary between patients and can 
change dramatically over time depending on disease progression and antibiotic exposure (Zhao et al., 2012). Despite profound inter-individual microbial community dynamics, strains of the opportunistic human pathogens, $P$. aeruginosa and $S$. aureus, are among the dominant inhabitants in the CF airways and have been co-isolated in both early-stage as well as chronic late-stage infections (Lording et al., 2006; Harrison, 2007; Fugere et al., 2014). In accordance, a recent cohort study at the Danish CF Centre reported that approximately $16 \%$ of the CF patients chronically infected with $S$. aureus were also chronically infected with $P$. aeruginosa (Dalboge et al., 2013). Interaction studies between these two bacterial species often use laboratory model strains of $P$. aeruginosa, which effectively kills co-cultured $S$. aureus by producing a range of antistaphylococcal molecules such as pyocyanin, LasA, LasB, rhamnolipids and the 4-hydroxy-2alkylquinoline (HAQ) molecule, 4-hydroxy-2-heptylquinoline- $\mathrm{N}$-oxide (HQNO) (Mashburn et al., 2005; Palmer et al., 2005; Hoffman et al., 2006; Mitchell et al., 2010; Korgaonkar and Whiteley, 2011; Korgaonkar et al., 2013; DeLeon et al., 2014). Therefore, limited investigations have addressed the potential effect of $S$. aureus on $P$. aeruginosa.

Important for $P$. aeruginosa pathogenesis is the hierarchical QS system, which is mediated by two chemically distinct classes of signaling molecules, the $N$-acylhomoserine lactones and the HAQ molecules (Pearson et al., 1997; Heeb et al., 2011). More than $50 \mathrm{HAQ}$ molecules divided into five distinct series (the series A-E congeners) have been identified from $P$. aeruginosa, where especially the signaling molecule 3,4-dihydroxy-2-heptylquinoline (PQS; the most prominent Series B congener) is important for regulating the production of virulence factors (Deziel et al., 2004; Heeb et al., 2011). However, within bacterial populations associated with long-term infections, loci encoding the QS system have been found to accumulate mutations, and can result in extensive metabolic and phenotypic modifications of host-adapted strains compared with strains of environmental origin (Smith et al., 2006; D'Argenio et al., 2007; Yang et al., 2011; Damkiaer et al., 2013), which may subsequently affect their interaction with other microorganisms. Thus, the outcome of interaction between $P$. aeruginosa and $S$. aureus may be strain dependent.

This work expands on our previous observation with a human host-adapted $P$. aeruginosa strain, DK2-P2M24-2003, from a dominant lineage, DK2, that have evolved through decades of growth in chronically infected Danish CF patients (Yang et al., 2011; Damkiaer et al., 2013; Marvig et al., 2013). P. aeruginosa DK2-P2M24-2003 showed a distinct colony morphology with centered autolysis and a metallic sheen coverage associated with the area of autolysis (Figure 1) (Michelsen et al., 2014). Notably, in contrast to $P$. aeruginosa PAO1, which is a widely used laboratory strain in microbial interaction

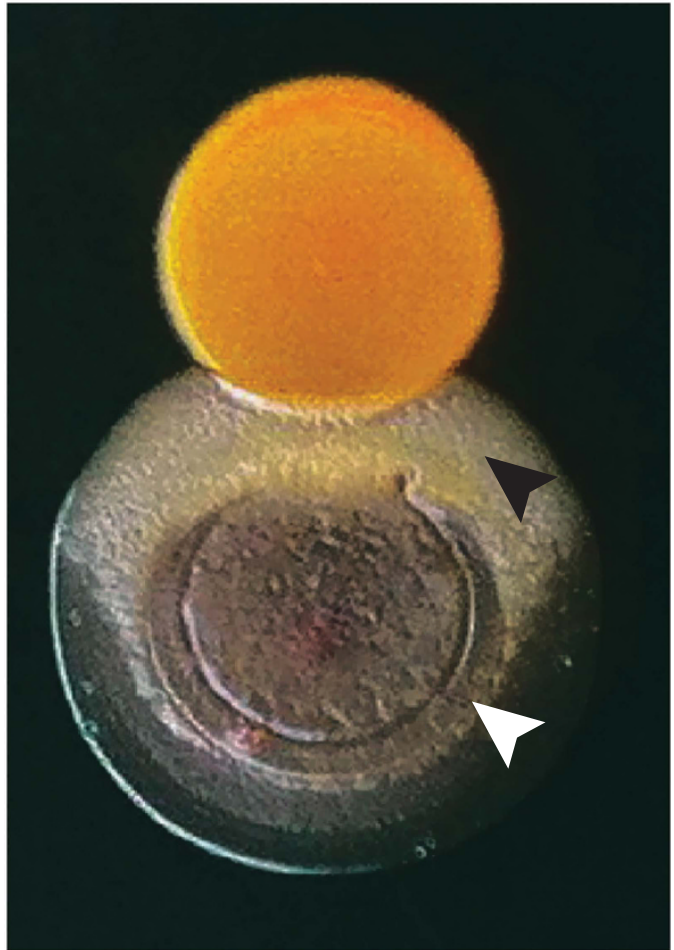

Figure 1 Co-culture of $P$. aeruginosa DK2-P24M2-2003 (lower colony) and $S$. aureus JE2 (upper colony). P. aeruginosa DK2P24M2-2003 show centered autolysis and a metallic sheen coverage associated with the lysed area (indicated by a white arrowhead). In interaction with $S$. aureus JE2, a thickened (white) $P$. aeruginosa colony morphology is observed at the interface (indicated by a black arrowhead).

studies (Hoffman et al., 2006; Mitchell et al., 2010; DeLeon et al., 2014; Phelan et al., 2014), $P$. aeruginosa DK2-P2M24-2003 did not suppress the growth of $S$. aureus USA300 strain JE2. Instead a thickened colony morphology appeared at the $P$. aeruginosa-S. aureus interface (Figure 1). Gfp expression measured from $P$. aeruginosa DK2P2M24-2003 tagged with a growth rate-dependent promoter:reporter gene construct, demonstrated an increased $P$. aeruginosa cell density at the area of thickened colony (Michelsen et al., 2014). These results suggested a commensal-like interaction, where $P$. aeruginosa DK2-P2M24-2003 benefits from the interaction with $S$. aureus.

The goal of this study was to provide insight into the metabolic divergence between $P$. aeruginosa DK2-P2M24-2003 and PAO1, which facilitates the commensal-like interaction between $P$. aeruginosa DK2-P2M24-2003 and $S$. aureus JE2. By combining molecular genetics with matrix-assisted laser desorption ionization-time of flight (MALDI-TOF) imaging mass spectrometry (IMS) on agar surfaces and mass spectral (MS) molecular network analysis on microbial extracts, a significant divergence in production of antimicrobial and HAQ molecules was detected between PAO1 and DK2-P2M24-2003. Such metabolic divergence may explain the different interaction patterns with $S$, aureus observed by 
PAO1 and DK2-P2M24-2003, respectively. Interestingly, the presence of $S$. aureus resulted in induction of a distinct HAQ profile in $P$. aeruginosa DK2P2M24-2003 during interaction, which significantly differed from the otherwise autolysing HAQ profile detected in the DK2-P2M24-2003 monoculture and in interaction with the $S$. aureus agrC mutant. Furthermore, a $P$. aeruginosa HAQ-mediated protection of $S$. aureus from the antibiotic effect of tobramycin was observed. We therefore propose a model, where the HAQ molecules produced by $P$. aeruginosa DK2-P2M24-2003 provide an important factor for the proto-cooperative interaction with $S$. aureus JE2, in which both bacterial species benefit from the relationship.

\section{Materials and methods}

Bacterial strains, plasmids and media

The bacterial strains and plasmids used in this study are described in Table 1. P. aeruginosa and $S$. aureus were routinely cultured in LB broth/agar. $P$. aeruginosa-S. aureus interaction assays were performed on tryptic soy broth (Sigma-Aldrich, Denmark A/S, Copenhagen, Denmark) supplemented with $1.2 \%$ (wt/vol) agar (TSA), LB with $1.2 \%$ agar (Sigma-Aldrich, Denmark A/S) or artificial sputum medium (ASM) (Kirchner et al., 2012) supplemented with $1.2 \%$ agar. All liquid cultures were incubated at $37^{\circ} \mathrm{C}$ with shaking (200 r.p.m.) unless other stated. Antibiotics were used in following concentrations: ampicillin, $100 \mu \mathrm{g} \mathrm{ml}^{-1}$; gentamicin, $60 \mu \mathrm{g} \mathrm{ml}^{-1}$ for
$P$. aeruginosa transposon and allelic replacement mutants; tetracycline, $50 \mu \mathrm{g} \mathrm{ml}^{-1}$ for $P$. aeruginosa with inserted lux reporter fusion.

P. aeruginosa-S. aureus interaction assays. Stationaryphase cultures of $S$. aureus (optical density at $600 \mathrm{~nm}$ (OD600) of $\sim 2$ ) and $P$. aeruginosa (OD600 1.5) cultured in LB were diluted to OD600 $\sim 0.2$ and streaked or added as spots onto dry agar plates as previously described (Michelsen et al., 2014). Briefly, in the cross-streak assay, one loopful of $P$. aeruginosa culture was first streaked in one direction on the plate and left to dry before the plate was cross-streaked with one loopful of the $S$. aureus culture. During spot inoculation, $5 \mu l$ of the $P$. aeruginosa culture was spotted first onto agar plates and after drying $5 \mu \mathrm{l}$ of the $S$. aureus culture was spotted with $5 \mathrm{~mm}$ distance from the $P$. aeruginosa spot. The experiments were carried out in triplicate, and the plates were incubated at $37^{\circ} \mathrm{C}$ under aerobic conditions or anaerobically in a Type B Vinyl Anaerobic Chamber (Coy Lab Products, Grass Lake, MI, USA) filled with an atmosphere of $95 \%$ (vol/vol) nitrogen and 5\% (vol/vol) hydrogen. After $24-72 \mathrm{~h}$ of incubation, the bacterial colonies touched the edge of each other with $<1 \mathrm{~mm}$ distance between them. The plates were inspected visually, and the interaction at the $P$. aeruginosa-S. aureus interface was recorded.

The interaction assay with tobramycin was carried out as described by Hoffman et al. (2006). Briefly, the diluted $S$. aureus culture was applied to the surface of LB agar without or with addition of tobramycin (that is,

Table 1 Strains and plasmids used in this study

\begin{tabular}{|c|c|c|}
\hline Strain or plasmid & Genotype & Reference \\
\hline \multicolumn{3}{|l|}{ Plasmids } \\
\hline pBT20 & $\mathrm{Gm}^{\mathrm{r}}$; Himar1 C9 transposon & Kulasekara et al. (2005) \\
\hline prK600 & $\mathrm{Cm}^{\mathrm{r}}$; ori ColE1 RK2-mob+ RK2-mob+ helper plasmid for conjugation & Kessler et al. (1992) \\
\hline pEXG2-PAO601 & $\begin{array}{l}\mathrm{Gm}^{\mathrm{r}} ; s a c B \text {, allelic exchange vector with } \\
P A O 601 \text { gene deletion fragment }\end{array}$ & This study \\
\hline pCTX::pqsA'-lux & $\begin{array}{l}\text { Tetr; Promoter region and part of pqsA fused to } \\
\text { luxCDABE inserted into the miniCTX-1 delivery plasmid }\end{array}$ & Fletcher et al. (2007) \\
\hline pHK-pqsH::lux & $\begin{array}{l}\text { Tetr }^{\mathrm{r}} \text { Promoter region and part of } p q s H \text { fused to luxCDABE } \\
\text { inserted into the miniCTX-1 delivery plasmid }\end{array}$ & This study \\
\hline \multicolumn{3}{|l|}{ E. coli } \\
\hline HB101 & recA thi pro leu hsdRM $; \mathrm{Sm}^{\mathrm{r}}$ & Kessler et al. (1992) \\
\hline \multicolumn{3}{|l|}{ P. aeruginosa } \\
\hline PAO1 & WT, laboratory model strain & Stover et al. (2000) \\
\hline PAO1 pqsH::lux & $\begin{array}{l}\text { PAO1 containing a copy of the } p q s H \text { promoter linked } \\
\text { to the luxCDABE genes in the chromosome }\end{array}$ & This study \\
\hline DK2-P2M24-2003 & P. aeruginosa DK2 isolate from 2003 & Michelsen et al. (2014) \\
\hline DK2 pqsA'-lux & $\begin{array}{l}\text { DK2-P2M24-2003 containing a copy of the pqsA promoter } \\
\text { linked to the luxCDABE genes in the chromosome }\end{array}$ & This study \\
\hline DK2 pqsH::lux & $\begin{array}{l}\text { DK2-P2M24-2003 containing a copy of the pqsH promoter } \\
\text { linked to the luxCDABE genes in the chromosome }\end{array}$ & This study \\
\hline DK2-Tn1 & Transposon deletion of $p q s B$ in DK2-P2M24-2003 & This study \\
\hline \multicolumn{3}{|l|}{ S. aureus } \\
\hline JE2 & Plasmid-cured derivative of USA300 (FPR3757) & Fey et al. (2013) \\
\hline agrC & JE2 containing a transposon deletion of $\operatorname{agrC}$ & Fey et al. (2013) \\
\hline
\end{tabular}


$0.5,1.0,1.25,1.5,1.75,2.0$ or $2.5 \mu \mathrm{g} \mathrm{ml}^{-1}$ ) using a drigalski spatula, and $5 \mu$ l of the diluted $P$. aeruginosa cultures were added as spots to the $S$. aureus lawn. The experiments were carried out in triplicate, and the plates were incubated for $24 \mathrm{~h}$ at $37^{\circ} \mathrm{C}$.

Construction of reporter fusions and bioluminescence measurements

The plasmid pCTX::pqsA'-lux (Fletcher et al., 2007) was used for the $p q s A$ transcriptional fusion experiment. For the pqsH::lux reporter fusion, a 389-bp fragment containing the intergenic region upstream of $p q s H$ was amplified from genomic DNA using Phusion polymerase (Thermo Scientific) with the primers PqsH_F-PstI (5'-GAGACTGCAGATCACGCT GCACCTCGTCGC-3') and pqsH_R-XhoI (5'-ACATCT CGAGGTCGGTGAGATGGCCGCACA-3') and ligated with T4 DNA ligase into pHK-CTX2-lux (Marvig et al., 2014) after double digestion of the PCR fragment and vector with the restriction enzymes XhoI and PstI. The resulting plasmid pHK-pqsH::lux or pCTX::pqsA'-lux was introduced into $P$. aeruginosa strain PAO1 or DK2-P2M24-2003, respectively by triparental mating (Trieucuot et al., 1993) using the helper strain E. coli HB101/pRK600.

Diluted stationary phase cultures of $P$. aeruginosa PAO1 or DK2-P2M24-2003 containing the pqsH::lux or pqsA'-lux reporter fusions were spot-inoculated together with diluted stationary phase cultures of $S$. aureus on agar plates as described above. After $24 \mathrm{~h}$ of incubation, luminescence was recorded continuously for $10 \mathrm{~s}$ with a photon camera using an Hamamatsu Argus 50 image processor (Unit One Electronics, Birkerod, Denmark) and Argus 50 Control Program vers. 4.25 (Hamamatsu Photonics K.K., Ballerup, Denmark). The experiments were carried out in triplicate and the Tukey's HSD test was used in conjunction with ANOVA in order to evaluate which means were significantly different from one another. The tests were carried out with $\mathrm{R}$ version 3.0.3 (R Development Core Team, 2009).

Screen of S. aureus or P. aeruginosa transposon mutant libraries

The full $S$. aureus Nebraska transposon mutant library derived from S. aureus JE2 (Fey et al., 2013) was screened in a spot-inoculation setup with $P$. aeruginosa DK2-P2M24-2003 as illustrated in Supplementary Figure S5, to detect mutant strains which no longer induced the increased growth activity phenotype in $P$. aeruginosa. The $S$. aureus mutant strains were cultured in $200 \mu \mathrm{LB}$ in 96-well microtiter plates (Nunc) and $P$. aeruginosa DK2P2M24-2003 in $50 \mathrm{ml} \mathrm{LB}$ over night $(\mathrm{O} / \mathrm{N})$ at $37^{\circ} \mathrm{C}$ with shaking. Cultures of the $S$. aureus mutant strains were applied at one time onto OmniTrays (Nunc) containing LB agar by using a sterile replicator. After drying, the $P$. aeruginosa culture was applied in spots next to the $S$. aureus colonies using a sterile replicator. After 24-72 h of incubation the plates were visually inspected and $S$. aureus mutant strains were selected for a second round of screening using cross-streak and spot-inoculated assay as described above. The screening and rescreening were repeated three times.

Transposon mutagenesis of $P$. aeruginosa DK2P2M24-2003 was performed with the himar1 C9 mariner transposon from pBT20 (Kulasekara et al., 2005) by triparental mating using the helper strain E. coli HB101/pRK600. Strain DK2-P2M24-2003 transconjugants with transposons integrated into the chromosome were selected on Pseudomonas isolation agar plates supplemented with gentamicin. Approximately 1500 mutants were screened for an impaired interaction with $S$. aureus JE2. The $P$. aeruginosa mutant strains were cultured in $200 \mu \mathrm{LB}$ in 96-well microtiter plates (Nunc) and $S$. aureus JE2 in $50 \mathrm{ml} \mathrm{LB}$ $\mathrm{O} / \mathrm{N}$ at $37^{\circ} \mathrm{C}$ with shaking and screened in a spotinoculation setup as described above for the $S$. aureus mutant library as illustrated in Supplementary Figure S4. For mapping transposon insertion junctions in the $P$. aeruginosa DK2-P2M24-2003 chromosome, we used arbitrary PCR (Caetano-Anolles, 1993). The PCR was performed in two rounds with two different sets of primers as described in Supplementary Table S1. The PCR products were purified using the NucleoSpin PCR clean-up kit (Macherey-Nagel $\mathrm{GmbH} \&$ Co, Düren, Germany), as described by the manufacturer, sequenced at LGC Genomics $\mathrm{GmbH}$ (Berlin, Germany), and compared with the Genbank DNA sequence database using blastn (Altschul et al., 1990) and the $P$. aeruginosa DK2 reference genome (Accession no.: CP003149).

Construction of PAO601 deletion by allelic exchange in P. aeruginosa DK2-P2M24-2003

The PAO601 deletion in P. aeruginosa DK2-P2M242003 was performed using complete allelic exchange with a pEXG2-based construct containing a PAO601 gene deletion fragment, pEXG2-PAO601, kindly provided by $M$. Whiteley (University of Texas at Austin, USA). The allelic replacement construct was transferred into $P$. aeruginosa DK2-P2M24-2003 by triparental mating using the helper strain E. coli HB101/pRK600. After incubation O/N, merodiploid $P$. aeruginosa was selected by plating the conjugation mixture on Pseudomonas isolation agar plates with gentamicin $(\mathrm{Gm})$ followed by incubation until colonies appeared. Colonies were restreaked on $6 \%$ (wt/vol) sucrose-low salt $(0.5 \% \mathrm{NaCl}) \mathrm{LB}$ agar plates. Sucrose-resistant/Gm-sensitive colonies were restreaked on $6 \%$ sucrose-low salt LB agar plates, screened for the presence of the mutated allele by PCR and verified by sequencing at LGC Genomics.

\section{MALDI-TOF imaging mass spectrometry}

$P$. aeruginosa was co-cultured with $S$. aureus using spot inoculation on thin TSA plates as described 
above (that is, $10 \mathrm{ml}$ of medium in a standard Petri dish resulting in a medium thickness of about $1.5 \mathrm{~mm}$ ). After $24-72 \mathrm{~h}$ of incubation, areas of agar media containing the microbial colonies were excised from the Petri dish and transferred onto the MALDI stainless steel target plate. The subsequent

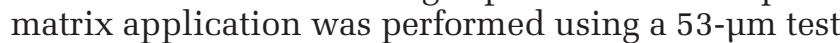
sieve whereby dry matrix was deposited evenly across the sample surface until the desired matrix thickness was achieved. The sample was dried $\mathrm{O} / \mathrm{N}$ followed by MALDI-TOF IMS analysis using a Bruker Microflex mass spectrometer. Data were collected from 50 to $3000 \mathrm{~m} / \mathrm{z}$ at $80 \mathrm{~Hz}$ laser frequency at a spatial resolution of $400 \mu \mathrm{m} \times 400 \mu \mathrm{m}$ using a Random Walk shot pattern. Data were analyzed using the Compass 1.2 software suite (FlexImaging 2.0, FlexControl 3.0 and FlexAnalysis 3.0; Bruker Daltonics, Ballerica, MA, USA) as previously described (Gonzalez et al., 2011). The resulting mass spectrum was binned at $0.5 \mathrm{~m} / \mathrm{z}$ increments and manually inspected for masses of interest. Correlating the spatial distribution of signals between the bacterial monocultures and the bacterial interactions allowed identification of signal intensities of masses ascribed to a specific bacterial source. Each mass was visualized by a specific false color superimposed onto an optical image of the original sample plate. The MALDI-TOF IMS analysis was carried out in triplicate and only masses that were observed in all sample replicates were investigated further.

LC-MS/MS analysis and construction of MS molecular network

For LC-MS/MS analysis, samples were diluted eightfold. Mass spectrometry was performed by using a Bruker Daltonics Maxis qTOF mass spectrometer equipped with a standard electrospray ionization source as previously described (Phelan et al., 2014). For accurate mass measurements, lock mass internal calibration used a wick saturated with hexakis(1H,1H,3H-tetrafluoropropoxy)phosphazene ions (Synquest Laboratories, Alachua, FL, USA; m/z 922.0098) located within the source.

Ions of interest observed in MALDI-TOF IMS (P. aeruginosa DK2-P2M24-2003-S. aureus interaction at $48 \mathrm{~h}$ ) were correlated to the collected MS/MS data on the corresponding extracts. Tandem mass spectra were converted into a MS/MS network and clustered according to the protocol described by Watrous et al. (2012) using the GNPS infrastructure (gnps.ucsd.edu) as MSV000078634, whereby each node is a cluster-consensus spectrum and each edge corresponds to a significant pair wise alignment. The MS molecular network was visualized in Cytoscape (Smoot et al., 2011). All algorithms assumed precursor mass tolerance of 1.0 and fragment mass tolerance of 0.5 Da. MS/MS-based dereplication was performed by incorporating accumulated reference spectra into the MS molecular network (Watrous et al., 2012; Yang et al., 2013).

\section{Results}

Pseudomonas aeruginosa DK2-P2M24-2003 show divergent metabolic and interspecies interaction profiles

The interactions between $P$. aeruginosa strains PAO1 or DK2-P2M24-2003 and S. aureus JE2 were studied by culturing the bacteria side-by-side on agar surfaces in high-cell-density spots, which previously have been used to study microbial interactions (Gonzalez et al., 2011; Antonic et al., 2013; Michelsen et al., 2014; Phelan et al., 2014). Distinct colony morphologies and interaction patterns were observed by $P$. aeruginosa strains PAO1 and DK2P2M24-2003, respectively, during co-culturing with $S$. aureus JE2 (Figure 2), which supported our previous observations (Michelsen et al., 2014). Whereas $P$. aeruginosa PAO1 developed a smooth, green-pigmented colony and inhibited the growth of S. aureus, DK2-P2M24-2003 instead showed a commensal-like interspecies interaction with a thickened colony morphology at the $P$. aeruginosa$S$. aureus interface (Figure 2a), which previously has been identified as an area of increased Pseudomonas cell density (Michelsen et al., 2014). Importantly, the distinct interaction patterns by $P$. aeruginosa DK2-P2M24-2003 and P. aeruginosa PAO1, respectively, were reproducible during culturing on the different agar media, LB, TSA and ASM, which mimics the nutrient conditions of natural CF sputum, as well as both under aerobic and anaerobic growth conditions (Figure 3a).

The spatial and temporal distribution of metabolites was analyzed in the side-by-side $P$. aeruginosa-S. aureus interactions on agar surfaces by MALDI-TOF IMS (Yang et al., 2009). Differences in distribution of $\mathrm{m} / \mathrm{z}$ signals between $P$. aeruginosa PAO1 and DK2P2M24-2003 were examined and revealed very divergent metabolic profiles (Figure 2b). Several $\mathrm{m} / \mathrm{z}$ signals were detected from $P$. aeruginosa PAO1, which were not present in $P$. aeruginosa DK2-P2M24-2003. These $\mathrm{m} / \mathrm{z}$ signals corresponded to the antimicrobials pyocyanin $(\mathrm{m} / \mathrm{z} 211,1)$ and different rhamnolipids (for example, represented by $\mathrm{m} / \mathrm{z} 673$, 2) (Figure 2b) that previously have been identified from $P$. aeruginosa PAO1 using MALDITOF IMS (Phelan et al., 2014). In addition, some unidentified $S$. aureus $\mathrm{m} / \mathrm{z}$ signals of $\mathrm{m} / \mathrm{z} 231$ (3) and $\mathrm{m} / \mathrm{z} 256$ (4) were observed with increased intensity at the interface with $P$. aeruginosa DK2-P2M24-2003 but not with $P$. aeruginosa PAO1 (Figure 2b), which could be due to the suppression of $S$. aureus by PAO1. Notable, the signaling HAQ molecule, PQS, and the antimicrobial HAQ molecule, HQNO, $(\mathrm{m} / \mathrm{z} 260,6)$, both of which were present in $P$. aeruginosa PAO1, were not detected in $P$. aeruginosa DK2-P2M24-2003 (Figure 2b). 


\begin{tabular}{|lccc|}
\hline $\begin{array}{l}\text { P. aeruginosa } \\
\text { strain }\end{array}$ & $\begin{array}{l}\text { Metallic sheen/ } \\
\text { autolysis }\end{array}$ & $\begin{array}{l}\text { Inhibition of } \\
\text { S. aureus }\end{array}$ & $\begin{array}{l}\text { Increased cell density } \\
\text { of } P \text {. aeruginosa }\end{array}$ \\
\hline PAO1 & - & + & - \\
DK2 & + & - & + \\
\hline
\end{tabular}

b

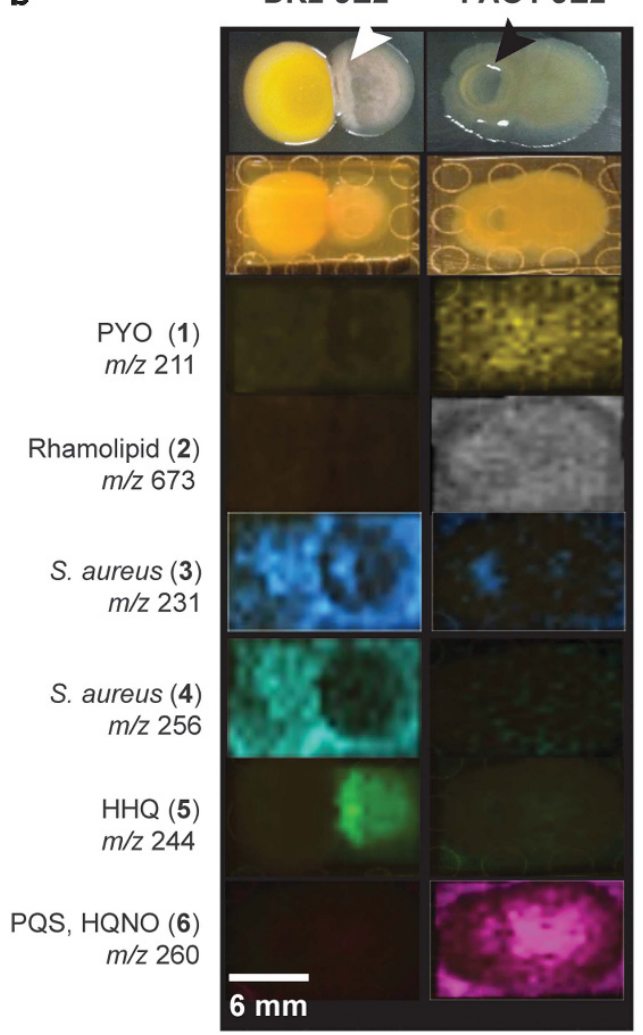

C

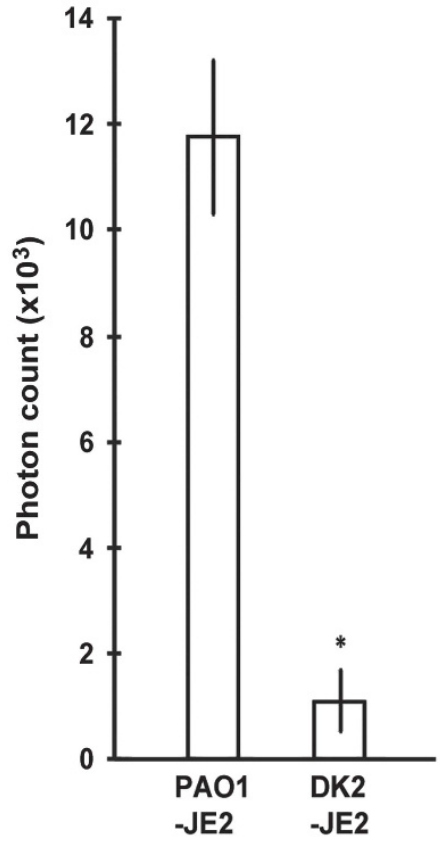

Figure 2 (a) Recorded phenotypes of P. aeruginosa PAO1 and DK2-P2M24-2003 in interaction with $S$. aureus JE2. Phenotypes were scored as: +: Yes or - : No. (b) P. aeruginosa (right colony)-S. aureus JE2 (left colony) interactions on agar surfaces with $P$. aeruginosa DK2-P2M242003 (DK2-JE2) or PAO1 (PAO1-JE2) co-cultured for $72 \mathrm{~h}$ on TSA. Black arrowhead indicates suppression of $S$. aureus and white arrowhead indicates the zone of thickened DK2-P2M24-2003 colony morphology. The $P$. aeruginosa-S. aureus interactions were transferred to a MALDI plate before MALDI-TOF IMS analysis (second row of pictures). Strain-specific $\mathrm{m} / \mathrm{z}$ signals in $P$. aeruginosa-S. aureus interactions were identified by MALDI-TOF IMS analysis and visualized by different colors. The $\mathrm{m} / \mathrm{z}$ distributions of the $P$. aeruginosa antimicrobial molecules, (1) pyocyanin (PYO) (yellow, $\mathrm{m} / \mathrm{z}$ 211) and (2) a representative example for the rhamnolipid family (gray, $\mathrm{m} / \mathrm{z} 673$ ), (3, 4) unidentified $S$. aureus molecules of $\mathrm{m} / \mathrm{z} 231$ (blue) and $\mathrm{m} / \mathrm{z} 256$ (cyan), respectively, and the $P$. aeruginosa HAQ molecules, (5) HHQ (green, $\mathrm{m} / \mathrm{z} 244$ ) and (6) PQS and HQNO (purple, $\mathrm{m} / \mathrm{z} 260$ ) are shown. The MALDI-TOF IMS spatial resolution was $500 \mu \mathrm{m}$, with a detection range of 50-3000 Da. (c) Bioluminescence measured by photon counting from P. aeruginosa PAO1 or DK2-P2M24-2003 (DK2) with a pqsH::lux reporter fusion in interaction $S$. aureus JE2. ${ }^{*} P<0.05$ by Tukey's HSD test compared with PAO1. Error bars represent s.d., $n=3$.

DK2-P2M24-2003 instead showed an increased production of 2-heptyl-4-hydroxyquinoline, HHQ $(\mathrm{m} / \mathrm{z} 244,5)$ (Figure 2b), the biosynthetic precursor of PQS (Deziel et al., 2004). PqsH catalyzes the conversion of HHQ to PQS in P. aeruginosa (Deziel et al., 2004). Thus, to investigate whether the lack of PQS production in $P$. aeruginosa DK2-P2M24-2003 was due to an altered expression of the pqsH gene, we constructed a luxCDABE-based pqsH promotor fusion (Becher and Schweizer, 2000), which was incorporated into the PAO1 and DK2-P2M24-2003 chromosome, respectively. Indeed, a significant decrease in $p q s H$ expression, determined by emitted bioluminescence, was observed by $P$. aeruginosa
DK2-P2M24-2003 compared with $P$. aeruginosa PAO1 (Figure 2c). Thus, given what is known about PQS and virulence factor production in P. aeruginosa (Deziel et al., 2004; Heeb et al., 2011), its loss could predict to facilitate co-existence of $P$. aeruginosa and $S$. aureus.

The presence of S. aureus JE2 induces a distinct $H A Q$ profile and altered colony morphology in P. aeruginosa DK2-P2M24-2003

Compared with the metabolic profile of $P$. aeruginosa DK2-P2M24-2003 spotted in monoculture, we noticed by MALDI-TOF IMS an increased intensity 
a

\begin{tabular}{|l|c|c|c|c|c|}
\hline \multirow{2}{*}{$\begin{array}{l}\text { P. aeruginosa } \\
\text { strains }\end{array}$} & \multicolumn{5}{|c|}{ Culture media/conditions } \\
\cline { 2 - 6 } & LB & TSA & ASM & aerobic & anaerobic \\
\hline PA01 & inh & inh & inh & inh & inh \\
\hline DK2 wt & + & + & + & + & + \\
\hline DK2-Tn1 & - & - & - & - & N/A \\
\hline DK2-PA0601 & + & + & + & + & N/A \\
\hline
\end{tabular}

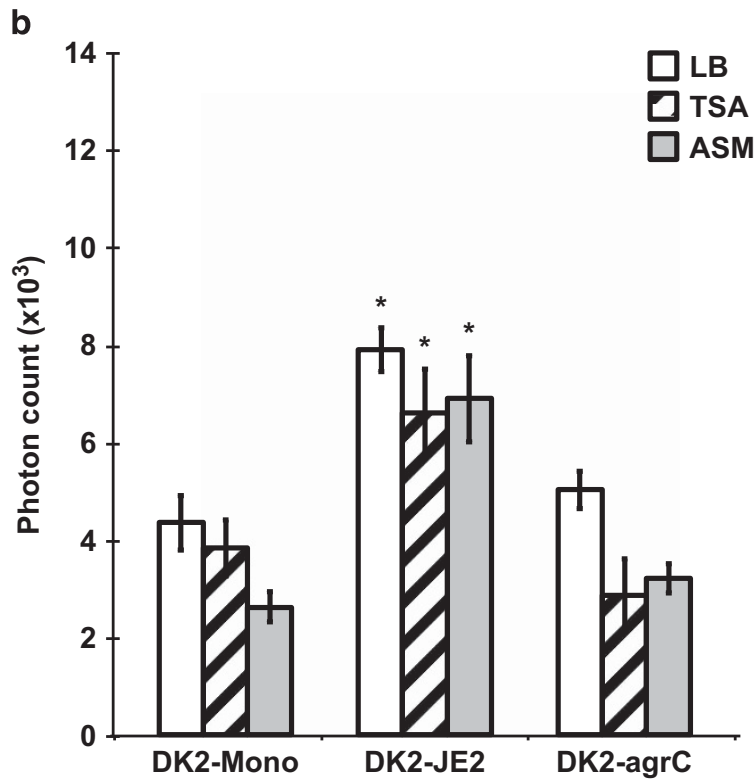

Figure 3 (a) Interactions between the different $P$. aeruginosa strains, PAO1, DK2-P2M24-2003 (DK2 wt), the DK2-P2M24-2003 pqsB mutant (DK2-Tn1) or the PAO601 mutant (DK2-PAO601) and S. aureus JE2 when cultured on different agar media, LB, TSA, ASM and under aerobic or anaerobic conditions, respectively. 'inh': inhibition of $S$. aureus growth by $P$. aeruginosa; ' + ': thickened $P$. aeruginosa colony morphology in interaction with $S$. aureus; '-': no thickened $P$. aeruginosa colony morphology; 'N/A': not analyzed. (b) Bioluminescence measured by photon counting from P. aeruginosa DK2-P2M24-2003 with a pqsA'-lux reporter fusion in monoculture (DK2-Mono), in interaction with S. aureus JE2 (DK2-JE2) or the agrC mutant (DK2-agrC) when cultured on LB, TSA or ASM agar surfaces, respectively. ${ }^{*} P<0.05$ by Tukey's HSD test compared with DK2-mono. Error bars represent s.d., $n=3$.

of at least five Pseudomonas-derived molecules with the signals $\mathrm{m} / \mathrm{z} 244,258,270,272$ and 298, respectively, at the $P$. aeruginosa DK2-P2M242003-S. aureus JE2 interface (Figure 4a). The increased intensity of the different molecules was most pronounced after 48 and $72 \mathrm{~h}$ of interaction with $S$. aureus JE2, and correlated with the area of thickened $P$. aeruginosa DK2-P2M24-2003 colony morphology (that is, increased cell density) (Figure 4a and Supplementary Figure S1).

To facilitate the identification of the molecules observed in MALDI-TOF IMS, organic extracts were prepared of a duplicate plate of the interacting $P$. aeruginosa DK2-P2M24-2003 and $S$. aureus colonies and were analyzed using LC-MS/MS. This enabled us to construct a MS/MS-based molecular network (Watrous et al., 2012) of the extracted $P$. aeruginosa and $S$. aureus molecules, which were visualized in Cytoscape (Smoot et al., 2011) (Supplementary Figure S2). In this network, the parent ions $(\mathrm{m} / \mathrm{z})$ are displayed as nodes and nodes with high MS/MS spectral similarity are clustered together representing the same chemical class of molecules. The MS molecular network analysis showed a majority of parent ions derived from the $P$. aeruginosa DK2-P2M24-2003 extracts, and by incorporating MS/MS data of known molecules (Watrous et al., 2012; Yang et al., 2013), a cluster with members of the quinolone family (HAQ molecules) was identified (Supplementary Figure S2). Five different series A and D congeners, HHQ, C8 HHQ, C9:db NHQ, NHQ and C11:db UHQ, respectively were annotated using spectral comparison and fragmentation analysis (Figure $4 \mathrm{~b}$ and Supplementary Figure S3), whereas the rest of the molecules within the cluster remained uncharacterized 


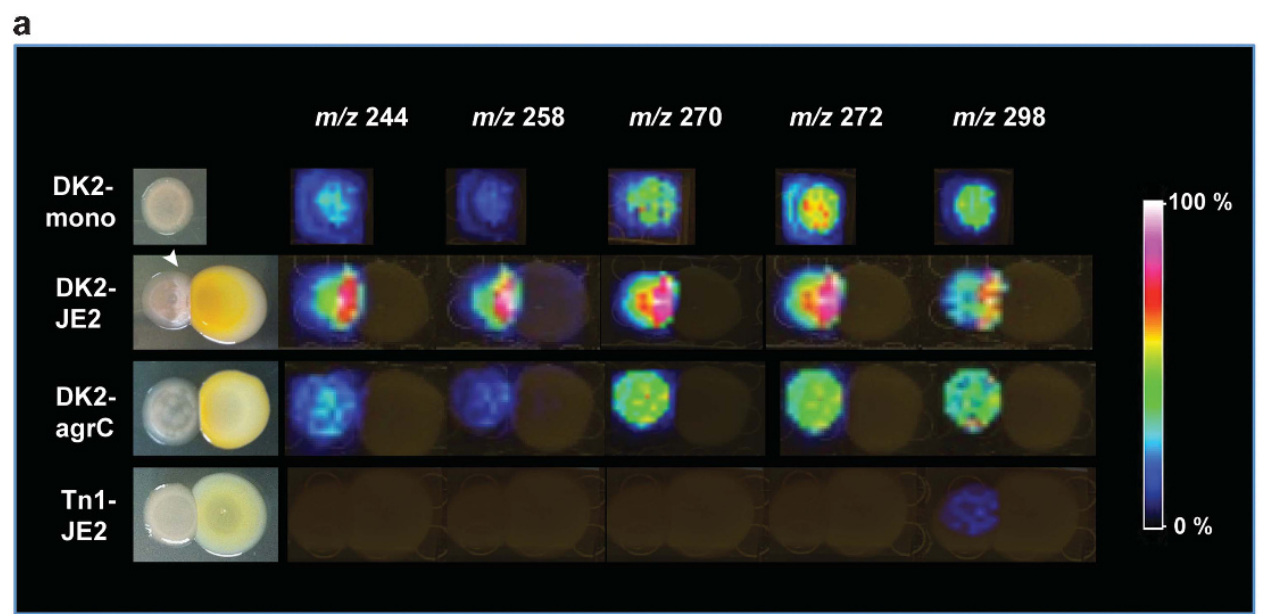

b
\begin{tabular}{|lllllll|}
\hline $\boldsymbol{m} / \boldsymbol{z}$ & Formula & Form & Name & $\mathbf{H}^{+}$Calc. & $\mathbf{H}^{+}$Obs & Series \\
244 & $\mathrm{C}_{16} \mathrm{H}_{21} \mathrm{NO}$ & $\mathrm{H}^{+}$ & $\mathrm{HHQ}$ & 244.1696 & 244.163 & $\mathrm{~A}$ \\
$258^{*}$ & $\mathrm{C}_{17} \mathrm{H}_{23} \mathrm{NO}$ & $\mathrm{H}^{+}$ & $\mathrm{C} 8 \mathrm{HHQ}$ & 258.385 & 258.185 & $\mathrm{~A}$ \\
270 & $\mathrm{C}_{18} \mathrm{H}_{23} \mathrm{NO}$ & $\mathrm{H}^{+}$ & $\mathrm{C} 9: \mathrm{db} \mathrm{NHQ}$ & 270.1853 & 270.177 & $\mathrm{D}$ \\
272 & $\mathrm{C}_{18} \mathrm{H}_{25} \mathrm{NO}$ & $\mathrm{H}^{+}$ & $\mathrm{NHQ}$ & 272.2009 & 272.193 & $\mathrm{~A}$ \\
298 & $\mathrm{C}_{20} \mathrm{H}_{27} \mathrm{NO}$ & $\mathrm{H}^{+}$ & $\mathrm{C} 11: \mathrm{db} \mathrm{UHQ}$ & 298.2166 & 298.207 & $\mathrm{D}$ \\
\hline
\end{tabular}

Figure 4 (a) MALDI-TOF IMS analysis of P. aeruginosa DK2-P2M24-2003 in monoculture (DK2-mono), in interaction with $S$. aureus JE2 (DK2-JE2) or in interaction with the $S$. aureus agrC mutant (DK2-agrC) as well as the P. aeruginosa DK2-P2M24-2003 pqsB mutant, Tn1, in interaction with $S$. aureus JE2 (Tn1-JE2) after $48 \mathrm{~h}$ of co-culturing. The $\mathrm{m} / \mathrm{z}$ distributions of $P$. aeruginosa molecules are displayed as false color overlays of an optical image. Ion-intensity color scaling indicates a highest production of molecules with $\mathrm{m} / \mathrm{z} 224,258,270,272$ and 298, respectively, at the interface between P. aeruginosa DK2-P2M24-2003 and S. aureus JE2. White arrowhead indicates the zone of thickened $P$. aeruginosa colony morphology. (b) HAQ molecules were annotated from the MS molecular network using MS/MS-based dereplication or fragmentation analysis (indicated by *). Nomenclature, calculated and observed $[\mathrm{M}+\mathrm{H}]^{+}$masses, as well as the HAQ series are shown for the different HAQ molecules according to previous studies (Deziel et al., 2004; Heeb et al., 2011).

(Supplementary Figure S2). The annotated HAQ molecules corresponded to the molecules detected with increased intensity in the MALDI-TOF IMS analysis of the $P$. aeruginosa DK2-P2M24-2003-S. aureus JE2 interaction (Figure 4). Similar to the observation from MALDI-TOF IMS, the signaling and antimicrobial HAQ molecules, PQS and HQNO (that is, $m / z$ 260), respectively were not detected nor identified using MS molecular networking on the $P$. aeruginosa DK2-P2M24-2003 extracts (Supplementary Figure S2).

The pqsABCDE operon is involved in the biosynthesis of HAQ molecules in P. aeruginosa (Deziel et al., 2004). Thus, in order to confirm an increased production of HAQ molecules by $P$. aeruginosa DK2P2M24-2003 during interaction with $S$. aureus JE2, we constructed a luxCDABE-based reporter gene fusion (Becher and Schweizer, 2000) with the promoter of $p q s A$. This construct was incorporated into the DK2-P2M24-2003 chromosome. By measuring the bioluminescence from $P$. aeruginosa DK2P2M24-2003, we observed an up to threefold increase in $p q s A$ expression in interaction with
S. aureus JE2 compared with DK2-P2M24-2003 in monoculture when analyzed on the same media (LB, TSA and ASM, respectively) on which the thickened Pseudomonas colony morphology was observed (Figure 3b). Taking together, these results indicated that the induced HAQ profile is important for the thickened $P$. aeruginosa DK2-P2M24-2003 colony morphology observed during interaction with S. aureus JE2.

Screen for mechanisms involved in the interaction between P. aeruginosa DK2-P2M24-2003 and S. aureus JE2

A knockout mutation in the gene, PAO601, which previously has been identified to be important for the sensing of $S$. aureus by $P$. aeruginosa (Korgaonkar et al., 2013), was constructed in P. aeruginosa DK2P2M24-2003, however, did not affect the interaction between DK2-P2M24-2003 and $S$. aureus JE2 (Figure 3a)

Therefore, we established a DK2-P2M24-2003 mariner-based transposon mutant library of 1500 
mutant strains and performed a genetic screen in a spot-interaction setup with $S$. aureus JE2 (Supplementary Figure S4), to further identify genes involved in the $P$. aeruginosa DK2-P2M24-2003 response to $S$. aureus JE2. Four distinct $P$. aeruginosa mutant strains, designated Tn1, Tn2, Tn3 and Tn4, respectively were found, showing no thickened colony morphology in interaction with $S$. aureus JE2 (Figure 4a and Supplementary Figure S4). These mutant strains had the transposon inserted into the $p q s B$ gene (Tn1), which is part of the PQS biosynthetic operon, pqsA-E (Heeb et al., 2011); a gene encoding a tryptophan permease (Tn2); a gene encoding a putative permease (Tn3); and a gene encoding a UvrD/REP helicase (Tn4), respectively (Supplementary Figure S4). In addition, the $P$. aeruginosa mutant Tn1 did not show the autolysing phenotype of the DK2-P2M24-2003 wild-type strain (Figure 4a and Supplementary Figure S4). MALDI-TOF IMS analysis of $P$. aeruginosa Tn1 revealed a defective production of HAQ molecules, likely because of the $p q s B$ gene knockout in this mutant (Figure 4a), which further supported an importance of the induced HAQ profile in DK2P2M24-2003 for the thickened colony morphology (that is, increased cell density) detected at the interface with $S$. aureus JE2.

In addition to the genetic screen of $P$. aeruginosa DK2-P2M24-2003, the 1920 mutants included in the S. aureus JE2 Nebraska Transposon Mutant Library (Fey et al., 2013) were screened in a similar spotinteraction setup with $P$. aeruginosa DK2-P2M242003 (Supplementary Figure S5). We identified nine distinct $S$. aureus mutants from this mutant screen, which did not induce the thickened colony morphology in $P$. aeruginosa DK2-P2M24-2003 (Supplementary Figure S5). These mutants were the previously identified strains with the transposon inserted into the agrC, agrB, $\operatorname{clpP}$ or sarA genes, respectively, (Michelsen et al., 2014) as well as $S$. aureus strains with transposons inserted in agrA (NE1532) or in genes encoding different membrane transporter proteins (mutants NE496, NE969 and NE1509, respectively) (Supplementary Figure S5). The molecular interaction between the $S$. aureus agrC mutant and $P$. aeruginosa DK2-P2M24-2003 was further investigated by MALDI-TOF IMS analysis. In contrast to the interaction with the $S$. aureus JE2 wild-type strain, no induction of HAQ molecules was detected in DK2-P2M24-2003 by the presence of the $S$. aureus agrC mutant, which instead resembled the autolysing HAQ profile of the DK2-P2M24-2003 monoculture (Figure 4a). In addition, the pqs $A$ expression profile in $P$. aeruginosa DK2-P2M24-2003 during interaction with $S$. aureus agrC was similar to that of DK2-P2M24-2003 in monoculture (Figure 3b), which suggests that a functional agr system in $S$. aureus is required for inducing the distinct HAQ profile and thickened colony morphology in $P$. aeruginosa DK2-P2M24-2003.
$H A Q$ molecules produced by $\mathrm{P}$. aeruginosa

DK2-P2M24-2003 protect S. aureus from the antibiotic effect of tobramycin

HQNO produced by $P$. aeruginosa have previously been attributed to protect $S$. aureus from killing by aminoglycosides such as tobramycin (Hoffman et al., 2006). While MALDI-TOF IMS and the MS molecular network analysis did not detect HQNO in $P$. aeruginosa DK2-P2M24-2003, we were curious whether DK2-P2M24-2003 was still able to protect $S$. aureus against tobramycin during co-culture. The effect of DK2-P2M24-2003 was compared with that of the $P$. aeruginosa DK2-P2M24-2003 pqsB mutant, Tn1 or $P$. aeruginosa $\mathrm{PAO} 1$, respectively. In the absence of tobramycin $P$. aeruginosa PAO1 suppressed the growth of $S$. aureus JE2, whereas no $S$. aureus inhibition was observed by the $P$. aeruginosa DK2-P2M24-2003 wild-type or Tn1 strain (Figure 5). In the presence of tobramycin levels above the minimal inhibitory concentration of $S$. aureus JE2 (MIC; $1.5 \mu \mathrm{g} \mathrm{ml}^{-1}$ ), however, we observed growth of $S$. aureus in a zone surrounding the HQNO producing $P$. aeruginosa PAO1 (Figure 5), indicating a protective effect of $S$. aureus by HQNO as previous described (Hoffman et al., 2006). Surprisingly, S. aureus growth was also detected in a zone surrounding $P$. aeruginosa DK2-P2M24-2003, which was not able to produce HQNO, whereas no growth was observed in the presence of $P$. aeruginosa $\mathrm{Tn} 1$, deficient in HAQ biosynthesis (Figure 5). These results suggested a more complex HAQ-mediated protection of $S$. aureus against the effect of tobramycin. The HAQ-mediated protection from P. aeruginosa DK2-P2M24-2003 was not induced exclusively by the presence of $S$. aureus JE2, as a similar protective effect was observed of the $S$. aureus agrC mutant (Supplementary Figure S6).

\section{Discussion}

The importance of understanding interspecies interactions in complex polymicrobial infections is manifested by enhanced antibiotic resistance or persistency of bacterial pathogens at the infection site. One example is the interaction between the opportunistic human pathogens $P$. aeruginosa and $S$. aureus that may cause more severe infections when found together compared with when present alone (Peters et al., 2012; Hubert et al., 2013; Pastar et al., 2013). However, the nature of long-term interactions during co-existence of these two species (as occurs in chronic polymicrobial infection) has proven difficult to study in vitro, due to $S$. aureus growth suppression by $P$. aeruginosa exoproducts (Duan et al., 2003; Hoffman et al., 2006; Mitchell et al., 2010; DeLeon et al., 2014). Here, we postulate differences in strains that form polymicrobial infections contribute to this. Accordingly, we observed an effective killing of $S$. aureus JE2 by the laboratory reference strain $P$. aeruginosa 


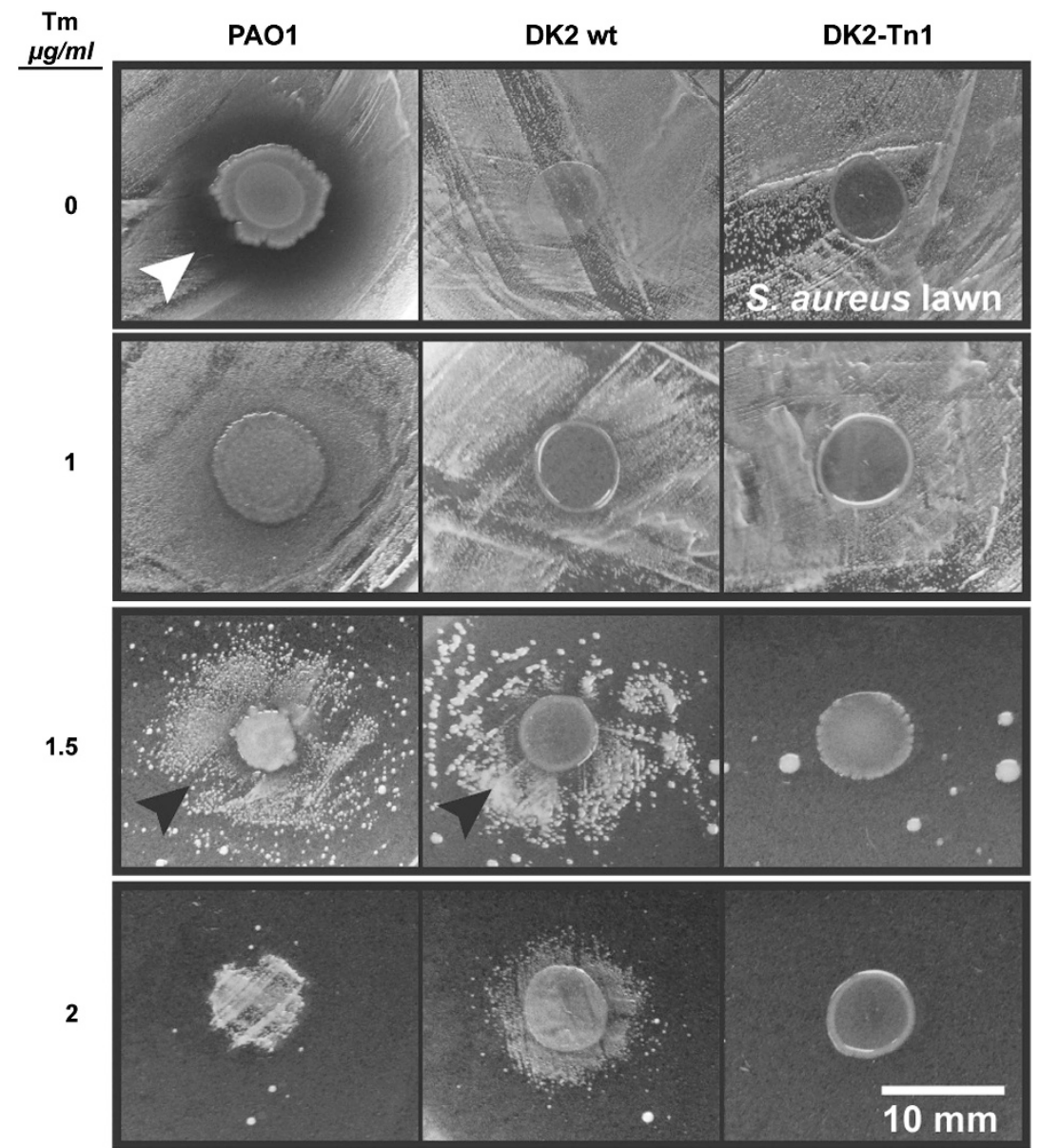

Figure 5 Colonies of $P$. aeruginosa PAO1, the DK2-P2M24-2003 wild type (DK2 wt) or the pqsB mutant, Tn1 (DK2-Tn1) grown on a lawn of $S$. aureus JE2 on LB agar plates with the addition of tobramycin (Tm) as indicated. P. aeruginosa PAO1 produced HQNO, whereas DK2P2M24-2003 and Tn1 were defective for HQNO or HAQ production, respectively. On LB agar without tobramycin P. aeruginosa PAO1, but not DK2-P2M24-2003 or Tn1, suppressed the growth of $S$. aureus (indicated by a white arrowhead). By tobramycin concentrations $>1.5 \mu \mathrm{g} \mathrm{ml} \mathrm{m}^{-1} \mathrm{~S}$. aureus JE2 growth was observed in a zone surrounding the $P$. aeruginosa PAO1 and DK2-P2M24-2003 colonies (indicated by black arrowheads), but not the Tn1 colony.

PAO1 when co-cultured on agar surfaces. However, in contrast to PAO1, suppression of $S$. aureus JE2 growth was not observed by the human host-adapted $P$. aeruginosa strain, DK2-P2M24-2003. This coexistence of DK2-P2M24-2003 and $S$. aureus JE2 was reproducible on different agar media, including ASM, and under aerobic and anaerobic growth conditions, which thereby allowed us to explore the molecular-genetic basis of co-culture interactions between these strains.

By combining molecular genetics with MALDITOF IMS and MS molecular networking, we observed a significant divergent metabolic profile between P. aeruginosa PAO1 and DK2-P2M24-2003. Although $P$. aeruginosa PAO1 produced pyocyanin, rhamnolipids and HQNO, which are all part of the antimicrobial circuitry of $P$. aeruginosa (Hoffman et al., 2006; Strateva and Mitov, 2011), these antimicrobials were not detected in $P$. aeruginosa DK2-P2M24-2003, and most likely explain why DK2P2M24-2003 and S. aureus JE2 are able to co-exist. It has been well established that $P$. aeruginosa evolves during long-term infections in human hosts (Smith et al., 2006; Marvig et al., 2013). Loss-of-function mutations in the lasR gene are commonly detected during $P$. aeruginosa adaptation to the $\mathrm{CF}$ airways (Smith et al., 2006; D'Argenio et al., 2007), which encodes a central quorum sensing-dependent transcriptional activator of multiple virulence genes (Gallagher et al., 2002; D'Argenio et al., 2007). In addition, LasR regulates the expression of $p q s H$, which is involved in the biosynthesis of HAQ molecules (that is, responsible for the conversion of the precursor HHQ to the signaling molecule, PQS; Deziel et al., 2004). Together with other regulatory mutations, the lasR loss-of-function mutation has been detected in the human host-adapted $P$. aeruginosa DK2 lineage (Yang et al., 2011; Damkiaer et al., 2013) and is also present in $P$. aeruginosa DK2-P2M24-2003 (Michelsen et al., 2014). Therefore, this mutation could serve as a contributing factor for the divergent metabolic profile observed in DK2-P2M24-2003 compared to PAO1. Besides LasR our previous study with a lasR 


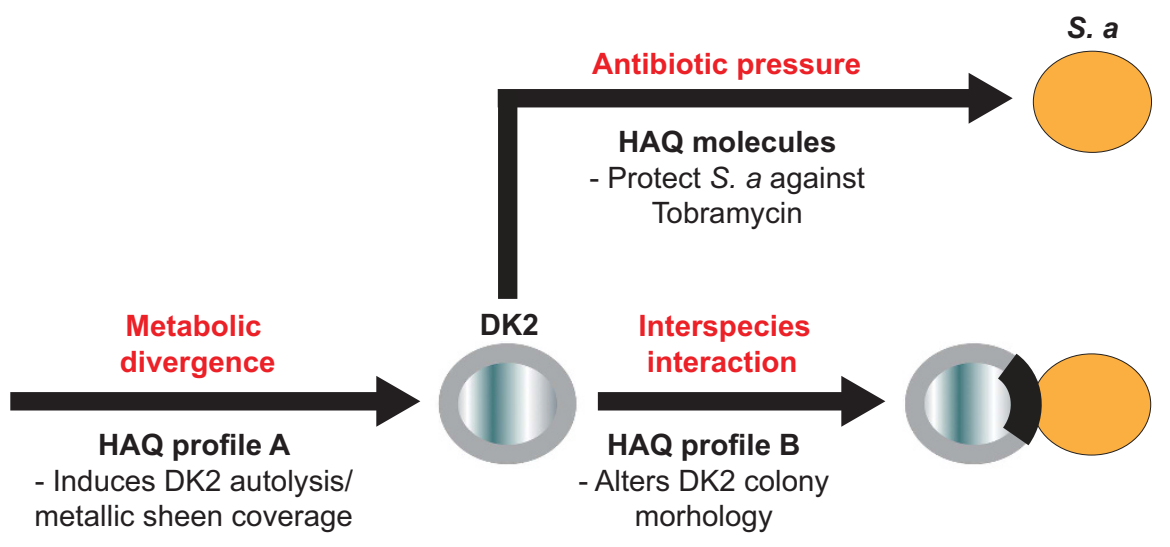

Legends:

P. aeruginosa DK2-P2M24-2003 (DK2)

Area of increased $P$. aeruginosa DK2 cell density

S. aureus JE2 (S. a)

Figure 6 Model for $P$. aeruginosa DK2-P2M24-2003-S. aureus JE2 proto-cooperation and the importance of the $P$. aeruginosa produced HAQ molecules for this interaction. According to the model, genetic adaptation during long-term infection is manifested by metabolic divergence in DK2-P2M24-2003 (DK2) resulting in an autolysing HAQ profile (HAQ profile A) and distinct colony morphology as well as the ability to co-exist with $S$. aureus (S. a.). In interaction with $S$. aureus, a thickened colony morphology (i.e., increased cell density) is observed in DK2-P2M24-2003 explained by an induction of a second HAQ profile (HAQ profile B). In addition, HAQ molecules produced by DK2-P2M24-2003 mediate a protection of $S$. aureus from the killing effect by tobramycin frequently used in CF therapy.

mutation in a PAO1 background clearly stated that additional mutations could contribute as well (Michelsen et al., 2014). A characteristic phenotype of $P$. aeruginosa lasR mutants is visible autolysis and an iridescent metallic sheen coverage of colonies (D'Argenio et al., 2007). This phenotype has been attributed to an increased ratio of 'Series A congeners' (for example, HHQ) to 'Series B congeners' (for example, PQS) of HAQ molecules (D'Argenio et al., 2007; Gupta and Schuster, 2012). Autolysis and metallic sheen coverage was also observed of the DK2-P2M24-2003 colony. However, besides an increased HHQ:PQS ratio, additional Series A and D congeners (NHQ, UHQ and derivatives, respectively) were detected by MALDI-TOF IMS and MS molecular network analysis, which suggest a more complex autolysing HAQ profile in this strain.

Previous work has reported that $P$. aeruginosa responds to the $S$. aureus cell wall components, peptidoglycan and GlcNAc, with an increased production of virulence factors, and thereby further suppressing $S$. aureus during interaction (Korgaonkar and Whiteley, 2011; Korgaonkar et al., 2013). A strikingly different response to $S$. aureus was observed by $P$. aeruginosa DK2-P2M24-2003 in this study. Instead of an increased production of virulence factors, the presence of $S$. aureus JE2 induced a distinct HAQ profile of accumulated series $A$ and D congeners in DK2-P2M24-2003, thereby substituting the autolysing HAQ profile at the
$P$. aeruginosa-S. aureus interface. Thus, the distinct HAQ profile in DK2-P2M24-2003 at the interface with $S$. aureus JE2 could explain the observed thickened colony morphology, which we have previously identified as a zone with increased Pseudomonas cell density (Michelsen et al., 2014). This model is summarized in Figure 6. Our model was further supported by a genetic screen of 1500 $P$. aeruginosa DK2-P2M24-2003 transposon mutants in interaction with $S$. aureus JE2. Here we identified two mutations, in a gene encoding a tryptophan permease and the $p q s B$ gene, respectively that no longer responded to $S$. aureus. Tryptophan provides a precursor for HAQ molecules (Farrow and Pesci, 2007; Heeb et al., 2011), whereas pqsB is directly involved in the biosynthesis of HAQs (Deziel et al., 2004; Heeb et al., 2011). The DK2-P2M24-2003 pqsB mutant (Tn1) showed a distinct smooth colony morphology with no autolysis, which could be explained by the abolished HAQ production and thereby no autolysing HAQ profile in this strain as revealed by MALDI-TOF IMS. In addition to the effect on $P$. aeruginosa itself, the HAQ molecules produced by DK2-P2M24-2003 were associated with a protection of $S$. aureus from killing by tobramycin, an antibiotic commonly used in CF treatments (Gibson et al., 2003). These results of reciprocal change in growth phenotype of both strains supported a model of proto-cooperation between P. aeruginosa DK2-P2M24-2003 and S. aureus JE2. 
It became clear that peptidoglycan and GlcNAc were not involved in the induced HAQ profile in $P$. aeruginosa DK2-P2M24-2003 by $S$. aureus JE2, since a knockout of the sensing mechanism, PAO601 (Korgaonkar et al., 2013), in P. aeruginosa DK2-P2M24-2003 did not eliminate the thickened colony morphology. The genetic background of $S$. aureus JE2 has been used as a host for creating a well-characterized sequence-defined transposon mutant library, that is the Nebraska Transposon Mutant Library (NTML), to investigate $S$. aureus biology and pathogenesis (Fey et al., 2013). We have previously identified the genes, $\operatorname{agr} C$, $\operatorname{agr} B$, clpP and sarA, in $S$. aureus JE2 to be involved in the induction of the DK2-P2M24-2003 thickened colony morphology (Michelsen et al., 2014). The involvement of these genes was also confirmed in this study by a genetic screen with the full $S$. aureus JE2 NTML consisting of 1952 mutant strains. MALDI-TOF IMS analysis of the interaction between DK2-P2M24-2003 and the $S$. aureus agrC mutant compared with $S$. aureus JE2 revealed no induction of the distinct HAQ profile in DK2-P2M24-2003, which could explain why the thickened Pseudomonas colony morphology was not observed in this interaction. Instead an autolysing HAQ profile resembling the profile in DK2-P2M24-2003 monoculture was found in the $S$. aureus agrC interaction. Our genetic screen identified additional membrane transporter proteins, suggesting further the involvement of $S$. aureus extracellular molecules as agents for HAQ profile modifications in $P$. aeruginosa.

In summary, by combining molecular genetics and MALDI-TOF IMS analysis we demonstrate here a difference in the production of antimicrobial and signaling (HAQ) molecules between the laboratory model strain, $P$. aeruginosa PAO1, and the human host-adapted strain, P. aeruginosa DK2-P2M24-2003. The metabolic divergence explains their distinct interaction patterns, that is, antagonistic versus commensal-like, respectively with $S$. aureus JE2. The HAQ profile in P. aeruginosa DK2-P2M24-2003 was further modulated during interaction with S. aureus JE2, which facilitated a proto-cooperative relationship between the two bacteria. Thus, our results emphasize the importance of HAQ molecules in this interspecies interaction (as summarized in Figure 6). In the context of long-term infections, our finding of strain diversity cautions against generalizing conclusions of interactions between bacterial pathogens, which are based on experimental models with one or a few selected strains.

\section{Conflict of Interest}

The authors declare no conflict of interest.

\section{Acknowledgements}

We thank Martin Saxtorp Bojer (University of Copenhagen, Denmark) for help with screening the $S$. aureus Nebraska mutant library and Sara Dyhrberg (Technical University of Denmark, Denmark) for help with creating and screening the $P$. aeruginosa DK2-P2M24-2003 mutant library, respectively. In addition, we thank Tal Luzzatto Knaan (University of California at San Diego, USA) for help with running the LC-MS/MS analysis. Morten Sommer (Technical University of Denmark, Denmark) is acknowledged for providing access to an anaerobic chamber. The PAO601 deletion plasmid was kindly provided by Justine Murray and Marvin Whiteley (University of Texas, USA), and the pCTX::pqsA'-lux reporter plasmid was kindly provided by Paul Williams (The University of Nottingham, UK). Funding for this study was provided by an operating grant from the Villum Foundation to LJ (VKR023113). The Novo Nordisk Foundation supported HKJ as a clinical research stipend. The Aase \& Ejnar Danielsens Foundation (ref. no. 10-001120) and the Augustinus Foundation (ref. no. 13-4656) are acknowledged for travel grants to CFM. We further acknowledge Bruker and NIH Grant GMS10RR029121 for the support of the shared instrumentation infrastructure that enabled this work.

\section{References}

Altschul SF, Gish W, Miller W, Myers EW, Lipman DJ. (1990). Basic local alignment search tool. J Mol Biol 215: 403-410.

Antonic V, Stojadinovic A, Zhang B, Izadjoo MJ, Alavi M. (2013). Pseudomonas aeruginosa induces pigment production and enhances virulence in a white phenotypic variant of Staphylococcus aureus. Infect Drug Resist 6: 175-186.

Becher A, Schweizer HP. (2000). Integration-proficient Pseudomonas aeruginosa vectors for isolation of single-copy chromosomal lacZ and lux gene fusions. Biotechniques 29: 948-950 952.

Caetano-Anolles G. (1993). Amplifying DNA with arbitrary oligonucleotide primers. PCR Methods Appl 3: 85-94.

D'Argenio DA, Wu MH, Hoffman LR, Kulasekara HD, Deziel E, Smith EE et al. (2007). Growth phenotypes of Pseudomonas aeruginosa lasR mutants adapted to the airways of cystic fibrosis patients. Mol Microbiol 64: 512-533.

Dalboge CS, Pressler T, Høiby N, Nielsen KG, Johansen HK. (2013). A cohort study of the Copenhagen CF Centre eradication strategy against Staphylococcus aureus in patients with CF. J Cyst Fibros 12: 42-48.

Damkiaer S, Yang L, Molin S, Jelsbak L. (2013). Evolutionary remodeling of global regulatory networks during long-term bacterial adaptation to human hosts. Proc Natl Acad Sci USA 110: 7766-7771.

DeLeon S, Clinton A, Fowler H, Everett J, Horswill AR, Rumbaugh KP. (2014). Synergistic interactions of Pseudomonas aeruginosa and Staphylococcus aureus in an in vitro wound model. Infect Immun 82: 4718-4728.

Deziel E, Lepine F, Milot S, He J, Mindrinos MN, Tompkins RG et al. (2004). Analysis of Pseudomonas aeruginosa 4-hydroxy-2-alkylquinolines (HAQs) reveals a role for 4-hydroxy-2-heptylquinoline in cell-to-cell communication. Proc Natl Acad Sci USA 101: 1339-1344.

Duan K, Dammel C, Stein J, Rabin H, Surette MG. (2003). Modulation of Pseudomonas aeruginosa gene expression 
by host microflora through interspecies communication. Mol Microbiol 50: 1477-1491.

Farrow JM 3rd, Pesci EC. (2007). Two distinct pathways supply anthranilate as a precursor of the Pseudomonas quinolone signal. J Bacteriol 189: 3425-3433.

Fey PD, Endres JL, Yajjala VK, Widhelm TJ, Boissy RJ, Bose JL et al. (2013). A Genetic resource for rapid and comprehensive phenotype screening of nonessential Staphylococcus aureus genes. Mbio 4: e00537.

Fletcher MP, Diggle SP, Crusz SA, Chhabra SR, Camara M, Williams P. (2007). A dual biosensor for 2-alkyl-4quinolone quorum-sensing signal molecules. Environ Microbiol 9: 2683-2693.

Fugere A, Lalonde Seguin D, Mitchell G, Deziel E, Dekimpe V, Cantin AM et al. (2014). Interspecific small molecule interactions between clinical Isolates of Pseudomonas aeruginosa and Staphylococcus aureus from adult cystic fibrosis patients. PloS ONE 9: e86705.

Gallagher LA, McKnight SL, Kuznetsova MS, Pesci EC, Manoil C. (2002). Functions required for extracellular quinolone signaling by Pseudomonas aeruginosa. J Bacteriol 184: 6472-6480.

Gibson RL, Burns JL, Ramsey BW. (2003). Pathophysiology and management of pulmonary infections in cystic fibrosis. Am J Respir Crit Care Med 168: 918-951.

Gonzalez DJ, Haste NM, Hollands A, Fleming TC, Hamby M, Pogliano K et al. (2011). Microbial competition between Bacillus subtilis and Staphylococcus aureus monitored by imaging mass spectrometry. Microbiology 157: 2485-2492.

Gupta R, Schuster M. (2012). Quorum sensing modulates colony morphology through alkyl quinolones in Pseudomonas aeruginosa. BMC Microbiol 12: 30.

Harrison F. (2007). Microbial ecology of the cystic fibrosis lung. Microbiology 153: 917-923.

Heeb S, Fletcher MP, Chhabra SR, Diggle SP, Williams P, Camara M. (2011). Quinolones: from antibiotics to autoinducers. FEMS Microbiol Rev 35: 247-274.

Hoffman LR, Deziel E, D'Argenio DA, Lepine F, Emerson J, McNamara S et al. (2006). Selection for Staphylococcus aureus small-colony variants due to growth in the presence of Pseudomonas aeruginosa. Proc Natl Acad Sci USA 103: 19890-19895.

Hubert D, Reglier-Poupet H, Sermet-Gaudelus I, Ferroni A, Le Bourgeois M, Burgel PR et al. (2013). Association between Staphylococcus aureus alone or combined with Pseudomonas aeruginosa and the clinical condition of patients with cystic fibrosis. J Cyst Fibros 12: 497-503.

Kessler B, Delorenzo V, Timmis KN. (1992). A general system to integrate lac $Z$ fusions into the chromosome of gram-negative eubacteria - regulation of the Pm promoter of the TOL plasmid studied with all controling elements in monocopy. Mol Gen Genet 233: 293-301.

Kirchner S, Fothergill JL, Wright EA, James CE, Mowat E, Winstanley C. (2012). Use of artificial sputum medium to test antibiotic efficacy against Pseudomonas aeruginosa in conditions more relevant to the cystic fibrosis lung. J Vis Exp; e-pub ahead of print 5 June 2012; doi:10.3791/3857.

Korgaonkar A, Trivedi U, Rumbaugh KP, Whiteley M. (2013). Community surveillance enhances Pseudomonas aeruginosa virulence during polymicrobial infection. Proc Natl Acad Sci USA 110: 1059-1064.

Korgaonkar AK, Whiteley M. (2011). Pseudomonas aeruginosa enhances production of an antimicrobial in response to N-acetylglucosamine and peptidoglycan. J Bacteriol 193: 909-917.

Kulasekara HD, Ventre I, Kulasekara BR, Lazdunski A, Filloux A, Lory S. (2005). A novel two-component system controls the expression of Pseudomonas aeruginosa fimbrial cup genes. Mol Microbiol 55: 368-380.

Lieberman TD, Michel JB, Aingaran M, Potter-Bynoe G, Roux D, Davis MR et al. (2011). Parallel bacterial evolution within multiple patients identifies candidate pathogenicity genes. Nat Genet 43: 1275-1280.

Lording A, McGaw J, Dalton A, Beal G, Everard M, Taylor CJ. (2006). Pulmonary infection in mild variant cystic fibrosis: implications for care. J Cyst Fibros 5: 101-104.

Markussen T, Marvig RL, Gomez-Lozano M, Aanaes K, Burleigh AE, Hoiby $\mathrm{N}$ et al. (2014). Environmental heterogeneity drives within-host diversification and evolution of Pseudomonas aeruginosa. Mbio 5: e01592-01514.

Marvig RL, Damkiaer S, Khademi SM, Markussen TM, Molin S, Jelsbak L. (2014). Within-host evolution of Pseudomonas aeruginosa reveals adaptation toward iron acquisition from hemoglobin. Mbio 5: e00966-00914.

Marvig RL, Johansen HK, Molin S, Jelsbak L. (2013). Genome analysis of a transmissible lineage of Pseudomonas aeruginosa reveals pathoadaptive mutations and distinct evolutionary paths of hypermutators. PLoS Genet 9: e1003741.

Mashburn LM, Jett AM, Akins DR, Whiteley M. (2005). Staphylococcus aureus serves as an iron source for Pseudomonas aeruginosa during in vivo coculture. J Bacteriol 187: 554-566.

Michelsen CF, Christensen AM, Bojer MS, Hoiby N, Ingmer H, Jelsbak L. (2014). Staphylococcus aureus alters growth activity, autolysis, and antibiotic tolerance in a human host-adapted Pseudomonas aeruginosa lineage. J Bacteriol 196: 3903-3911.

Mitchell G, Seguin DL, Asselin AE, Deziel E, Cantin AM, Frost EH et al. (2010). Staphylococcus aureus sigma B-dependent emergence of small-colony variants and biofilm production following exposure to Pseudomonas aeruginosa 4-hydroxy-2-heptylquinoline- $N$ oxide. BMC Microbiol 10: 33.

Moree WJ, Phelan VV, Wu CH, Bandeira N, Cornett DS, Duggan BM et al. (2012). Interkingdom metabolic transformations captured by microbial imaging mass spectrometry. Proc Natl Acad Sci USA 109: 13811-13816.

Palmer KL, Mashburn LM, Singh PK, Whiteley M. (2005). Cystic fibrosis sputum supports growth and cues key aspects of Pseudomonas aeruginosa physiology. J Bacteriol 187: 5267-5277.

Pastar I, Nusbaum AG, Gil J, Patel SB, Chen J, Valdes J et al. (2013). Interactions of methicillin resistant Staphylococcus aureus USA300 and Pseudomonas aeruginosa in polymicrobial wound infection. PloS ONE 8: e56846.

Pearson JP, Pesci EC, Iglewski BH. (1997). Roles of Pseudomonas aeruginosa las and rhl quorum-sensing systems in control of elastase and rhamnolipid biosynthesis genes. J Bacteriol 179: 5756-5767.

Peters BM, Jabra-Rizk MA, O'May GA, Costerton JW, Shirtliff ME. (2012). Polymicrobial interactions: Impact on pathogenesis and human disease. Clin Microbiol Rev 25: 193-213.

Phelan VV, Moree WJ, Aguilar J, Cornett DS, Koumoutsi A, Noble SM et al. (2014). Impact of a transposon insertion in phzF2 on the specialized metabolite 
production and interkingdom interactions of Pseudomonas aeruginosa. J Bacteriol 196: 1683-1693.

Short FL, Murdoch SL, Ryan RP. (2014). Polybacterial human disease: the ills of social networking. Trends Microbiol 22: 508-516.

Smith EE, Buckley DG, Wu ZN, Saenphimmachak C, Hoffman LR, D'Argenio DA et al. (2006). Genetic adaptation by Pseudomonas aeruginosa to the airways of cystic fibrosis patients. Proc Natl Acad Sci USA 103: 8487-8492.

Smoot ME, Ono K, Ruscheinski J, Wang PL, Ideker T. (2011). Cytoscape 2.8: new features for data integration and network visualization. Bioinformatics 27: 431-432.

Stover CK, Pham XQ, Erwin AL, Mizoguchi SD, Warrener P, Hickey MJ et al. (2000). Complete genome sequence of Pseudomonas aeruginosa PAO1, an opportunistic pathogen. Nature 406: 959-964.

Strateva T, Mitov I. (2011). Contribution of an arsenal of virulence factors to pathogenesis of Pseudomonas aeruginosa infections. Ann Microbiol 61: 717-732.

R Development Core Team (2009). R: A Language and Environment for Statistical Computing. R Foundation for Statistical Computing: Vienna, Austria.

Trieucuot P, Derlot E, Courvalin P. (1993). Enhanced conjugative transfer of plasmid DNA from Escherichia coli to Staphylococcus aureus and Listeria monocytogenes. FEMS Microbiol Lett 109: 19-24.

Vega NM, Allison KR, Samuels AN, Klempner MS, Collins JJ. (2013). Salmonella typhimurium intercepts Escherichia coli signaling to enhance antibiotic tolerance. Proc Natl Acad Sci USA 110: 14420-14425.
Watrous J, Roach P, Alexandrov T, Heath BS, Yang JY, Kersten RD et al. (2012). Mass spectral molecular networking of living microbial colonies. Proc Natl Acad Sci USA 109: E1743-E1752.

Yang JY, Sanchez LM, Rath CM, Liu X, Boudreau PD, Bruns $\mathrm{N}$ et al. (2013). Molecular networking as a dereplication strategy. J Nat Prod 76: 1686-1699.

Yang L, Jelsbak L, Marvig RL, Damkiaer S, Workman CT, Rau MH et al. (2011). Evolutionary dynamics of bacteria in a human host environment. Proc Natl Acad Sci USA 108: 7481-7486.

Yang YL, Xu YQ, Straight P, Dorrestein PC. (2009). Translating metabolic exchange with imaging mass spectrometry. Nat Chem Biol 5: 885-887.

Zhao J, Schloss PD, Kalikin LM, Carmody LA, Foster BK, Petrosino JF et al. (2012). Decade-long bacterial community dynamics in cystic fibrosis airways. Proc Natl Acad Sci USA 109: 5809-5814.

(c) $(1) \Theta$ This work is licensed under a Creative Commons Attribution-NonCommercialNoDerivs 4.0 International License. The images or other third party material in this article are included in the article's Creative Commons license, unless indicated otherwise in the credit line; if the material is not included under the Creative Commons license, users will need to obtain permission from the license holder to reproduce the material. To view a copy of this license, visit http://creativecommons.org/ licenses/by-nc-nd/4.0/

Supplementary Information accompanies this paper on The ISME Journal website (http://www.nature.com/ismej) 